\title{
Accurate and Efficient Computation of High Order Zernike Moments
}

\author{
Gholam Reza Amayeh, Ali Erol, George Bebis, and Mircea Nicolescu \\ Computer Vision Laboratory, University of Nevada, Reno, NV 89557 \\ \{amayeh, aerol, bebis, mircea\}@cse.unr.edu
}

\begin{abstract}
Zernike Moments are useful tools in pattern recognition and image analysis due to their orthogonality and rotation invariance property. However, direct computation of these moments is very expensive, limiting their use especially at high orders. There has been some efforts to reduce the computational cost by employing quantized polar coordinate systems, which also reduces the accuracy of the moments. In this paper, we propose an efficient algorithm to accurately calculate Zernike moments at high orders. To preserve accuracy, we do not use any form of coordinate transformation and employ arbitrary precision arithmetic. The computational complexity is reduced by detecting the common terms in Zernike moments with different order and repetition. Experimental results show that our method is more accurate than the other methods and it has comparable computational complexity especially in case of using large images and high order moments.
\end{abstract}

\section{Introduction}

Moment functions of image intensity values are used to capture global features of the image in pattern recognition and image analysis [1]. Among many moment based descriptors, Zernike moments have minimal redundancy (due to the orthogonality of basis functions [2]), rotation invariance and robustness to noise; therefore they are used in a wide range of applications on image analysis, reconstruction and recognition [3]. However, there are also some technical difficulties in the calculation of Zernike moments due to the very high computational complexity and lack of numerical precision. It is usually not possible to calculate them accurately in reasonable time when the desired moment order is high and/or the images to be processed are large.

Little attention has been paid to the efficient and accurate calculation of Zernike moments [4-6]. Mukundan et al. [4] proposed a recursive algorithm for computing the Zernike and Legendre moments in polar coordinates. Belkasim et al [5] introduced another recursive algorithm using radial and angular expansions of Zernike orthonormal polynomials. Finally in a more recent study, Gu et al. [6] employed the square to circular transformation of Mukundan et al. [4] and more efficient recursive relations to develop an even faster algorithm but its accuracy is still limited to that of [4] because of approximate coordinate transformation. 
In this paper, we propose an algorithm to reduce computation cost of Zernike moments without sacrificing accuracy. To preserve accuracy we do not use any form of coordinate transformations and employ an arbitrary precision arithmetic library [7]. The computational complexity is reduced by computing the common terms in Zernike moments with different order and repetition only once.

In the next section, we briefly summarize the definition of Zernike moments. Then in section 3, we present our method. In section 4, the accuracy and computational complexity of our method is compared against other algorithms. Section 5 concludes the study.

\section{Zernike Moments}

Zernike moments are based on a set of complex polynomials that form a complete orthogonal set over the interior of the unit circle [8]. Zernike moments are defined to be the projection of the image function on these orthogonal basis functions. The basis functions $V_{n, m}(x, y)$ are given by

$$
V_{n, m}(x, y)=V_{n, m}(\rho, \theta)=R_{n, m}(\rho) e^{j m \theta}
$$

where $n$ is a non-negative integer, $m$ is non-zero integer subject to the constraints $n-|m|$ is even and $|m|<n, \rho$ is the length of the vector from origin to $(x, y), \theta$ is the angle between vector $\rho$ and the $x$-axis in a counter clockwise direction and $R_{n, m}(\rho)$ is the Zernike radial polynomial. The Zernike radial polynomials, $R_{n, m}(\rho)$, are defined as:

$$
R_{n, m}(\rho)=\sum_{k=|m|, n-k=\text { even }}^{n} \frac{(-1)^{\frac{n-k}{2}} \frac{n+k}{2} !}{\frac{n-k}{2} ! \frac{k+m}{2} ! \frac{k-m}{2} !} \rho^{k}=\sum_{k=|m|, n-k=\text { even }}^{n} \beta_{n, m, k} \rho^{k}
$$

Note that $R_{n, m}(\rho)=R_{n,-m}(\rho)$. The basis functions in equation 1 are orthogonal thus satisfy

$$
\frac{n+1}{\pi} \iint_{x^{2}+y^{2} \leq 1} V_{n, m}(x, y) V_{p, q}^{*}(x, y)=\delta_{n, p} \delta_{m, q}
$$

where

$$
\delta_{a, b}= \begin{cases}1 & a=b \\ 0 & \text { otherwise }\end{cases}
$$

The Zernike moment of order $n$ with repetition $m$ for a digital image function $f(x, y)$ is given by $[9]$

$$
Z_{n, m}=\frac{n+1}{\pi} \sum_{x^{2}+y^{2} \leq 1} f(x, y) V_{n, m}^{*}(x, y)
$$

where $V_{n, m}^{*}(x, y)$ is the complex conjugate of $V_{n, m}(x, y)$. To compute the Zernike moments of a given image, the image center of mass is taken to be the origin. The 
function $f(x, y)$ can then be reconstructed by the following truncated expansion [9]:

$$
\tilde{f}(x, y)=\sum_{n=0}^{N} \frac{C_{n, 0}}{2} R_{n, 0}(\rho)+\sum_{n=1}^{N} \sum_{m>0}\left(C_{n, m} \cos m \theta+S_{n, m} \sin m \theta\right) R_{n, m}(\rho)
$$

where $N$ is the maximum order of Zernike moments we want to use, $C_{n, m}$ and $S_{n, m}$ denote the real and imaginary parts of $Z_{n, m}$ respectively.

\section{Proposed Algorithm}

A way to improve the speed of Zernike moment calculation is to use a quantized polar coordinate system. In [4] and [6], a square to a circle transformation is utilized for this purpose. In [5], for a $M \times M$ image the angles are quantized to $4 M$ and radii are quantized to $M$ levels. A side effect of quantization is that some error is introduced especially in high order Zernike moments. In our method we avoid using any quantization, therefore, it's as accurate as the classical method. We obtain speed-up by detecting common terms in Zernike moments.

By substituting equations 2 and 1 in 5 and re-organizing the terms the Zernike moments can be calculated in the following form:

$$
\begin{array}{r}
Z_{n, m}=\frac{n+1}{\pi} \sum_{x^{2}+y^{2} \leq 1}\left(\sum_{k=|m|}^{n} \beta_{n, m, k} \rho^{k}\right) e^{-j m \theta} f(x, y) \\
=\frac{n+1}{\pi} \sum_{k=|m|}^{n} \beta_{n, m, k}\left(\sum_{x^{2}+y^{2} \leq 1} e^{-j m \theta} \rho^{k} f(x, y)\right) \\
=\frac{n+1}{\pi} \sum_{k=|m|}^{n} \beta_{n, m, k} \chi_{m, k}
\end{array}
$$

The $\chi_{m, k}$ 's defined in the equation 7 become a common term in the computation of Zernike moments with the same repetition as shown in Figure 1 for the case of repetition $m=0$. In general, to compute Zernike moments up to order $\mathrm{N}$, we need to compute $\chi_{m, k}$ for each repetition as demonstrated in Table 1 . The table shows all the $\chi_{m, k}$ to be computed for each repetition up to order 10. The second row of the table corresponds to the $\chi_{m, k}$ shown in Figure 1. Once all the entries in the table 1 are computed, Zernike moments with any order and repetition can be calculated as a linear combination of $\chi_{m, k}$ as shown in equation 7. Also note that the coefficients $\beta_{n, m, k}$ does not depend on the image or the coordinates; therefore, they are stored on a small lookup table to save further computation.

Another important issue in high order Zernike moment computation is numerical precision. Depending on the image size and the maximum order, double 


$$
\begin{aligned}
& Z_{0,0}=\beta_{0,0,0} \chi_{0,0} \\
& Z_{2,0}=\beta_{2,0,0} \chi_{0,0}+\beta_{2,0,2} \chi_{0,2} \\
& Z_{4,0}=\beta_{4,0,0} \chi_{0,0}+\beta_{4,0,2} \chi_{0,2}+\beta_{4,0,4} \chi_{0,4} \\
& Z_{6,0}=\beta_{6,0,0} \mid \chi_{0,0}+\beta_{6,0,2} \chi_{0,2}+\beta_{6,0,4} \chi_{0,4}+\beta_{6,0,6} \chi_{0,6} \\
& Z_{8,0}=\beta_{8,0,0} \chi_{0,0}+\beta_{8,0,2} \chi_{0,2}+\beta_{8,0,4} \chi_{0,4}+\beta_{8,0,6} \chi_{0,6}+\beta_{8,0,8} \chi_{0,8} \\
& Z_{10,0}=\beta_{10,0,0} \chi_{0,0}+\beta_{10,0,2} \chi_{0,2}+\beta_{10,0,4} \chi_{0,4}+\beta_{10,0,6} \chi_{0,6}+\beta_{10,0,8} \chi_{0,8}+\beta_{10,0,10} \chi_{0,10}
\end{aligned}
$$

Fig. 1. The common terms to compute Zernike moments up to 10 orders with zero repetition.

Table 1. $\chi_{m, k}$ 's needed to compute Zernike moments up to 10 order and $m$ repetition.

\begin{tabular}{|c|c|}
\hline repetition $m$ & $\chi_{m, k}$ \\
\hline 0 & $\chi_{0,0}, \chi_{0,2}, \chi_{0,4}, \chi_{0,6}, \chi_{0,8}, \chi_{0,10}$ \\
\hline 1 & $\chi_{1,1}, \chi_{1,3}, \chi_{1,5}, \chi_{1,7}, \chi_{1,9}$ \\
\hline 2 & $\chi_{2,2}, \chi_{2,4}, \chi_{2,6}, \chi_{2,8}, \chi_{2,10}$ \\
\hline 3 & $\chi_{3,3}, \chi_{3,5}, \chi_{3,7}, \chi_{3,9}$ \\
\hline 4 & $\chi_{4,4}, \chi_{4,6}, \chi_{4,8}, \chi_{4,10}$ \\
\hline 5 & $\chi_{5,5}, \chi_{5,7}, \chi_{5,9}$ \\
\hline 6 & $\chi_{6,6}, \chi_{6,8}, \chi_{6,10}$ \\
\hline 7 & $\chi_{7,7}, \chi_{7,9}$ \\
\hline 8 & $\chi_{8,8}, \chi_{8,10}$ \\
\hline 9 & $\chi_{9,9}$ \\
\hline 10 & $\chi_{10,10}$ \\
\hline
\end{tabular}

precision arithmetic does not provide enough precision. This fact is demonstrated in table 2, which shows the magnitude of the difference between Zernike moments computed using double precision and arbitrary precision arithmetic for a $300 \times 300$ image up to order 50 . It can be seen that the error becomes more and more significant with increasing order and decreasing repetition. Figure 3 shows the effect of this error on the orthogonality of basis functions. It can be clearly seen that in Figure 2(a), which is obtained using the double precision, equation 3 is violated to a great extent while Figure 2(b) which is obtained using arbitrary precision, the orthogonality is preserved.

To calculate the computational complexity of our algorithm, let the size of the image be $M \times M$ pixels, and maximum order of Zernike moments be $N$. At the beginning we need $M^{2} N$ multiplication to compute $\rho^{k} f(x, y)$ for $k=0,1, \ldots, N$ for once. Note that for $k=0$ we don't need any multiplication. In the next step, we must compute $\chi_{m, k}=\sum_{x} \sum_{y} e^{-j m \theta} \rho^{k} f(x, y)$. The number of $\chi_{m, k}$ to compute Zernike moments up to $N($ even $)$ order is $\frac{N}{2}\left(\frac{N}{2}+1\right)$. As there is no need for any multiplication for $m=0$ and $\chi_{m, k}$ is a complex number, this step requires $M^{2} N\left(\frac{N}{2}+1\right)$ multiplications and $2\left(M^{2}-1\right)\left(\frac{N}{2}+1\right)^{2}$ additions. For 
Table 2. The difference between magnitude of Zernike moments computed by classical method using double precision and Big Number class variables.

\begin{tabular}{|c|c|c|c|c|c|c|c|c|c|c|c|c|c|}
\hline Order, repetition & 0 & 2 & 4 & 6 & 8 & 10 & $\ldots$ & 40 & 42 & 44 & 46 & 48 & 50 \\
\hline 42 & $7.28 \mathrm{e}-4$ & $6.60 \mathrm{e}-4$ & $1.91 \mathrm{e}-4$ & $2.72 \mathrm{e}-4$ & $1.72 \mathrm{e}-4$ & $6.54 \mathrm{e}-6$ & $\ldots$ & $1.17 \mathrm{e}-17$ & $3.82 \mathrm{e}-17$ & & & & \\
\hline 44 & $3.50 \mathrm{e}-3$ & $5.57 \mathrm{e}-3$ & $1.11 \mathrm{e}-3$ & $1.18 \mathrm{e}-3$ & $1.05 \mathrm{e}-4$ & $1.49 \mathrm{e}-4$ & $\ldots$ & $1.52 \mathrm{e}-15$ & $1.30 \mathrm{e}-17$ & $1.04 \mathrm{e}-17$ & & & \\
\hline 46 & $3.97 \mathrm{e}-1$ & $6.48 \mathrm{e}-3$ & $5.25 \mathrm{e}-3$ & $2.04 \mathrm{e}-3$ & $2.57 \mathrm{e}-3$ & $1.07 \mathrm{e}-3$ & $\ldots$ & $2.12 \mathrm{e}-14$ & $1.48 \mathrm{e}-15$ & $9.06 \mathrm{e}-17$ & $2.60 \mathrm{e}-18$ & & \\
\hline 48 & $1.86 \mathrm{e} 0$ & $6.91 \mathrm{e}-2$ & $4.39 \mathrm{e}-2$ & $2.83 \mathrm{e}-2$ & $1.66 \mathrm{e}-2$ & $3.50 \mathrm{e}-3$ & $\ldots 5$ & $5.23 \mathrm{e}-14$ & $5.92 \mathrm{e}-14$ & $3.11 \mathrm{e}-16$ & $1.20 \mathrm{e}-16$ & $3.47 \mathrm{e}-18$ & \\
\hline 50 & $1.38 \mathrm{e} 1$ & $1.81 \mathrm{e} 0$ & $1.06 \mathrm{e}-1$ & $9.39 \mathrm{e}-2$ & $6.92 \mathrm{e}-2$ & $7.12 \mathrm{e}-2$ & $\ldots$ & $7.52 \mathrm{e}-12$ & $2.67 \mathrm{e}-13$ & $1.60 \mathrm{e}-14$ & $8.60 \mathrm{e}-16$ & $4.65 \mathrm{e}-17$ & $2.17 \mathrm{e}-1 \varepsilon$ \\
\hline
\end{tabular}

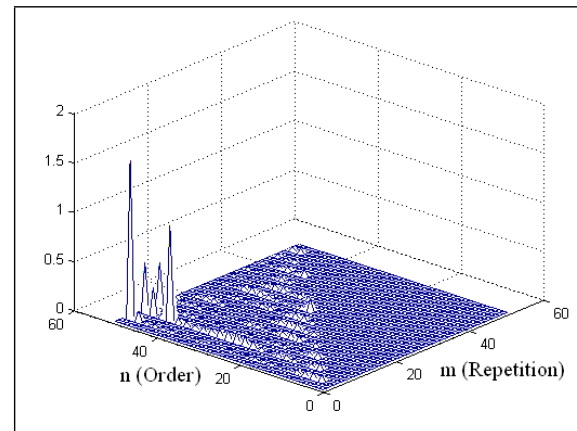

(a)

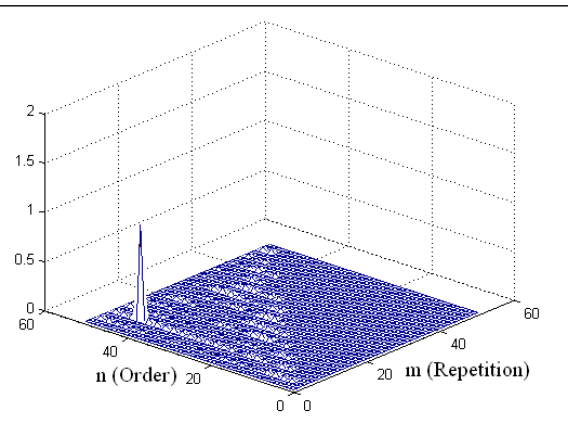

(b)

Fig. 2. Dot product of basis function with $n=43, m=7$ and other basis functions up to order 50 using (a) double precision and (b) arbitrary precision arithmetic.

large $N$ and $M$ the number of multiplications and additions to compute $Z_{n, m}$ according equation 7 is negligible.

\section{Experimental Results}

We compare the accuracy of the existing algorithms $[4,6,5]$ and our algorithm based on the fidelity of reconstruction. The test image that we used in our experiments is shown in Figure 3. This is a $64 \times 64$ image and Zernike moments up to order 40 are utilized for reconstruction. Figures 4(a), 4(b) and 4(c) show the results of Mukundan's [4], Gu's [6] and our method respectively. It can be seen that the former two algorithms give poor reconstructions mainly because of the square to circle transformation. The effect of the transformation is clearly visible in the reconstructed images.

The reconstruction result of Belkasim's [5] method using Zernike moments up to order 60 is shown in Figure 5(a). We used arbitrary precision arithmetic in the implementation of Belkasim's method as well. Our method's output is shown in Figure 5(b). It is possible to see that the Belkasim's method introduces some distortions at the edges. Our method's output is smoother in general. To make the difference more clear we computed reconstruction errors at different orders for the two algorithms. The error is computed using: 


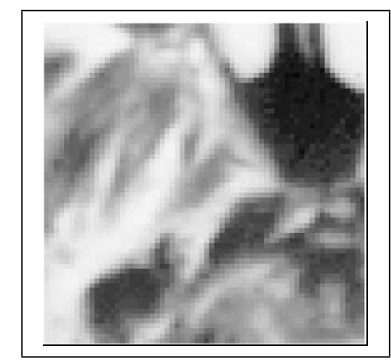

Fig. 3. Original gray level image.

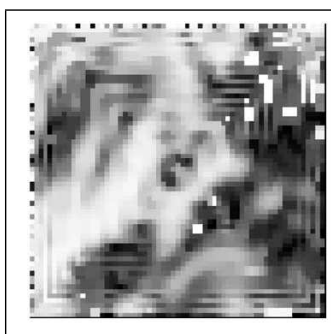

a

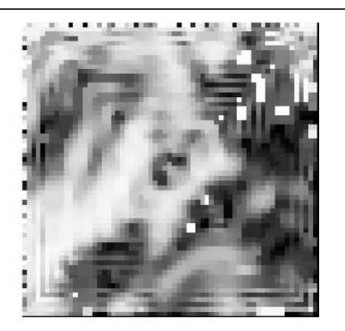

b

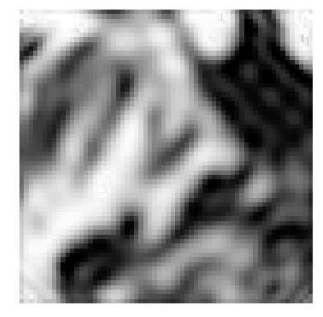

C

Fig. 4. The reconstructed images using the moment of order up to 40 by (a) Mukundan's method, (b) Gu's method and (c) our method.

$$
\varepsilon_{r}=\frac{\sum_{x} \sum_{y}|\tilde{f}(x, y)-f(x, y)|^{2}}{\sum_{x} \sum_{y} f(x, y)^{2}}
$$

where $f(x, y)$ is original image function and $\tilde{f}(x, y)$ is the reconstructed image. Table 3 shows the results of error computation. We would expect the error to decrease with the increasing order and our method (See column 1) behaves as expected; however, the behaivour of Belkasim's method is quite different, which shows that the quantization of polar coordinates has its effect at mainly higher order moments.

Table 3. Reconstruction error of Figure 3 by our method and Belkasim's method.

\begin{tabular}{|c|c|c|}
\hline Order & Our method & Belkasim's method \\
\hline 35 & 0.0647 & 0.0648 \\
\hline 40 & 0.0621 & 0.0628 \\
\hline 45 & 0.0596 & 0.063 \\
\hline 50 & 0.0370 & 0.0557 \\
\hline 55 & 0.0203 & 0.0645 \\
\hline 60 & 0.0133 & 0.0665 \\
\hline
\end{tabular}




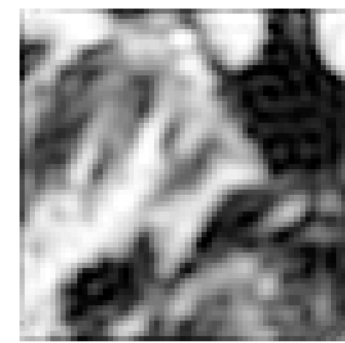

(a)

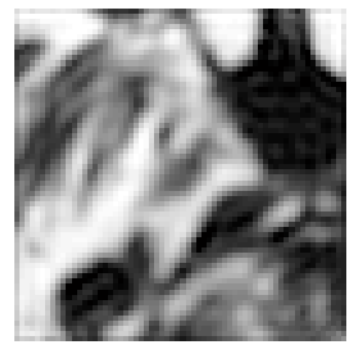

(b)

Fig. 5. The reconstructed images using the moment of order up to 60 by (a) Belkasim's method and (b) our method.

High order Zernike moments are used when there is a need to capture the details of the image. Figure 6 demonstrates this behavior using a $300 \times 300$ binary logo shown at the top left corner of the figure. Other images show reconstruction results using different orders. The reconstructed images up to order 20 only contains a rough silhouette of the wolf. In reconstructions up to order 50 , it is possible to see the head of wolf. At order 50, the head of the wolf is clearly visible but letters in the logo are still blurred. At order 70 it becomes possible to see the letters clearly.

Table 4 shows the number of multiplication and addition needed in our method and the others for $M \times M$ image using moments up to order $N$. It is clear that our method is not the fastest method; however, it is not extremely slow either. In terms number of multiplications our method stands close to Belkasim's method for large images $(M \gg 1)$ and high order moments $(N \gg 1)$.

Table 4. The comparison of the computational complexity of different methods.

\begin{tabular}{ccc}
\hline & Number of Addition & Number of Multiplication \\
\hline Mukundan's method & $\frac{N^{2} M^{2}}{2}+\frac{1}{8} N M^{3}$ & $2 N^{2}+N^{2} M^{2}+\frac{1}{4} M N^{3}$ \\
Belkasim's method & $N(M+2)(M-1)$ & $\frac{N^{2} M^{2}}{2}+2 M N$ \\
Gu's method & $\frac{3}{8} N^{2} M+2 N M^{2}+\frac{1}{12} N^{3} M+\frac{1}{4} N^{2} M^{2}$ & $\frac{N^{2} M}{2}+2 M^{2} N$ \\
Our method & $2\left(\frac{N}{2}+1\right)^{2}\left(M^{2}-1\right)$ & $\frac{N^{2} M^{2}}{2}+2 M^{2} N$ \\
\hline
\end{tabular}




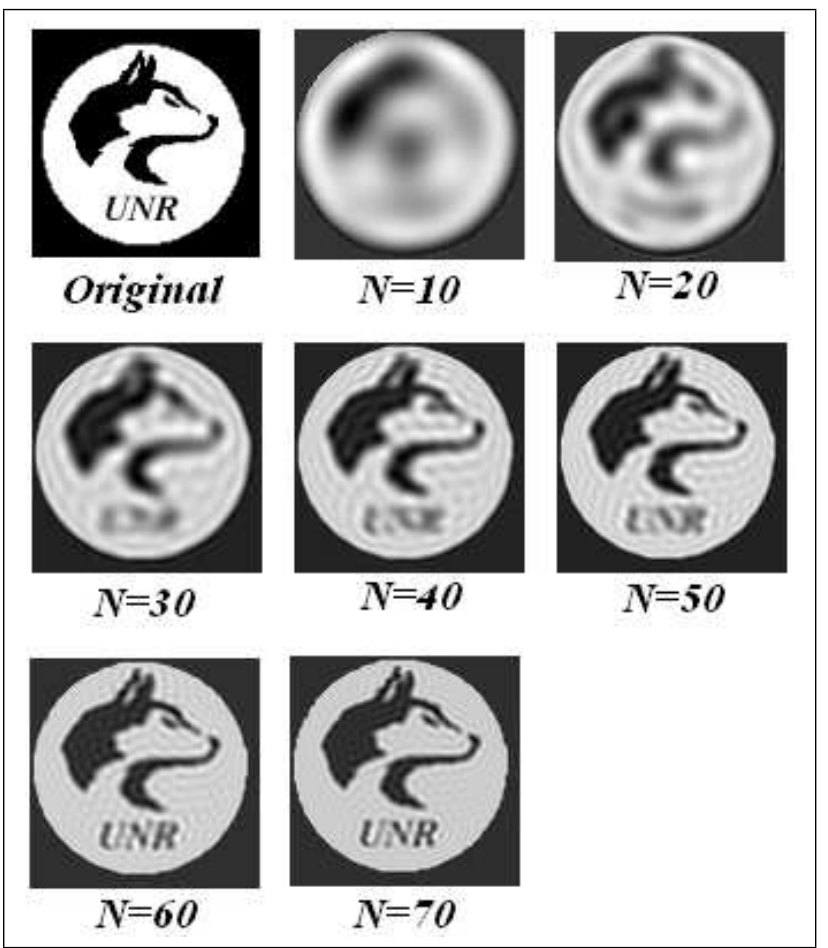

Fig. 6. Original image and reconstructions using different orders of Zernike moments.

\section{Conclusion}

We designed and implemented an accurate and efficient algorithm for high order Zernike moment calculation. We were able to compute very high order Zernike moments with reasonable computational complexity while preserving accuracy. According to the definition of Zernike moments for a digital image (See Equation 5 ) our computation is exact. The computational efficiency is provided by detection of common terms in Zernike moments. In our experimental results we also pointed out that polar coordinate quantization and double precision arithmetic are important sources of error in high order Zernike moment calculation.

\section{Acknowledgments}

This work was supported by NASA under grant \# NCC5-583.

\section{References}

1. Prokop, R.J., Reeves, A.P.: A survey of moment based techniques for unoccluded object representation. Graph. Models Image ProcessCVGIP Vol. 54,No. 5 (1992) 438460. 
2. Teague, M.R.: Image analysis via the general theory of moments. J. Opt. Soc. Am. Vol. 70,Issue. 8 (1980) 920930.

3. Teh, C.H., Chin, R.T.: On image analysis by the method of moments. IEEE Trans. Pattern Anal. Mach. Intell. Vol. 10 (1988) 485513.

4. Mukundan, R., Ramakrishnan, K.: Fast computation of legendre and zernike moments. Pattern Recognition Vol. 28,No. 9 (1995) 14331442.

5. Belkasim, S.O., Ahmadi, M., Shridhar, M.: Efficient algorithm for fast computation of zernike moments. IEEE 39th Midwest symposium on Circuits and Systems Vol. 3 (18-21 Aug. 1996) 1401 - 1404.

6. Gu, J., Shua, H.Z., Toumoulinb, C., Luoa, L.M.: A novel algorithm for fast computation of zernike moments. Pattern Recognition Vol. 35 (2002) 29052911.

7. GMP: (GNU multiple precision arithmetic library) http://www.swox.com/gmp.

8. Zernike, F.: Physica. (1934)

9. Khotanzad, A., Hong, Y.H.: Invariant image recognition by zernike moments. IEEE Trans. on Pattern Anal. and Machine Intell. Vol. 12 (1990) 489 - 498. 